\title{
THE BRANCHING CHANNEL NETWORK IN THE YANGTZE ESTUARY
}

\author{
Z.B. Wang ${ }^{1}$ and P.X. Ding ${ }^{2}$
}

\begin{abstract}
The channels in the Yangtze Estuary have an ordered-branching structure: The estuary is first divided by the Chongming Island into the North Branch and the South Branch. Then the South Branch is divided into the North Channel and South Channel by the Islands Changxing and Hengsha. The South Channel is again divided into the North and South Passage by the Jiuduansha Shoal. This three-level bifurcation and four-outlet configuration appears to be a natural character of the estuary, also in the past (Chen et al., 1982), although the whole system has been extending into the East China Sea in the southeast direction due to the abundant sediment supply from the Yangtze River. Recently, the natural development of the system seems to be substantially disturbed by human interferences, especially the Deep Navigation Channel Project. For the understanding of the behaviour of the bifurcating channel system in the estuary we present analysis on two aspects: (1) the equilibrium configuration of river delta distributary networks, and (2) influence of tidal flow on the morphological equilibrium of rivers. Based on the analyses we conclude that the branching channel structure of the Yangtze Estuary can be classified as tide-influenced river delta distributary networks. Its basic structure is the same as in case of river dominated delta. The empirical relations describing the basic features of the river-dominated distributary delta networks can be explained by theoretical analysis, although they are not fully satisfied by the Yangtze Estuary because of the influence of the tide. Two major influences of the tide are identified, viz. increasing the resistance to the river flow into the sea and increasing the sediment transport capacity. As consequence of these two influences the cross-sectional area of the river/estuary increases in the seawards direction and the bed slope decreases. The insights from the analyses are helpful for the understanding of the impact of the Deep Navigation Channel Project on the large scale morphological development of the estuary.
\end{abstract}

Keywords: Yangtze Estuary; River delta; Chanel network, Tide, morphological equilibrium

\section{INTRODUCTION}

The channels in the Yangtze Estuary have an ordered-branching structure (Figure 1): The estuary is first divided by the Chongming Island into the North Branch and the South Branch. Then the South Branch is divided into the North Channel and South Channel by the Changxing Island and the Hengsha Island. The South Channel is again divided into the North Passage and the South Passage by the Jiuduansha Shoal which is now developing into an island. This three-level bifurcation and fouroutlet configuration appears to be a natural character of the estuary, also in the past (Chen et al., 1982), although the whole system has been extending into the East China Sea in the southeast direction due to the abundant sediment supply from the Yangtze River. Each time the most upstream bifurcation disappears because of the closure of the most northern outlet and the largest island in the estuary joins the northern shore. At the same time a new bifurcation starts to develop dividing the most southern outlet into two outlets. With the geological data a number of this repeating process has been reconstructed (Chen et al., 1982). Each bifurcation in the Yangtze Estuary has a life cycle with its genesis, developing phase, deceasing phase and finally disappearance. The bifurcating channel system in the Yangtze Estuary shows typical characteristics and systematic development. However, no theoretical explanation has been given for the typical characteristics and the systematic development so far.

Recently the natural development of the system as described above seems to be substantially disturbed by human interferences, especially the Deep Navigation Channel Project (DNCP). The DNCP is meant to improve the navigability of the Yangtze Estuary through the North Passage, the northern channel of the most downstream bifurcation. The channel through the North Passage has been deepened from about $6 \mathrm{~m}$ to $12.5 \mathrm{~m}$ by building two guide bunds of about $50 \mathrm{~km}$ long and a series of groins (Figure 1) in combination with intensified dredging. First, artificially maintaining the North Passage open can be considered as a disturbance for the natural development on the long-term in which the northern channel tends to disappear eventually. Second, the recent observations indicate strong influence of the project on the morphological development of the system. Before the project the North Passage, used as a navigation route since 1983, was the main channel of this bifurcating system. At present the two passages have changed role: the South Passage has now become the main

\footnotetext{
${ }^{1}$ Deltares \& Delft University of Technology, Faculty of Civil Engineering and Geosciences, P.O. Box 177, 2600 MH Delft, The Netherlands

${ }^{2}$ SKLEC,East China Normal University, Shanghai, China
} 
channel for transporting water and sediment. This worrying development started after the second phase of the project when the two guide bunds were extended seaward to their present length. It looks with the second phase of the project a critical point has been passed beyond which adverse effects take place.

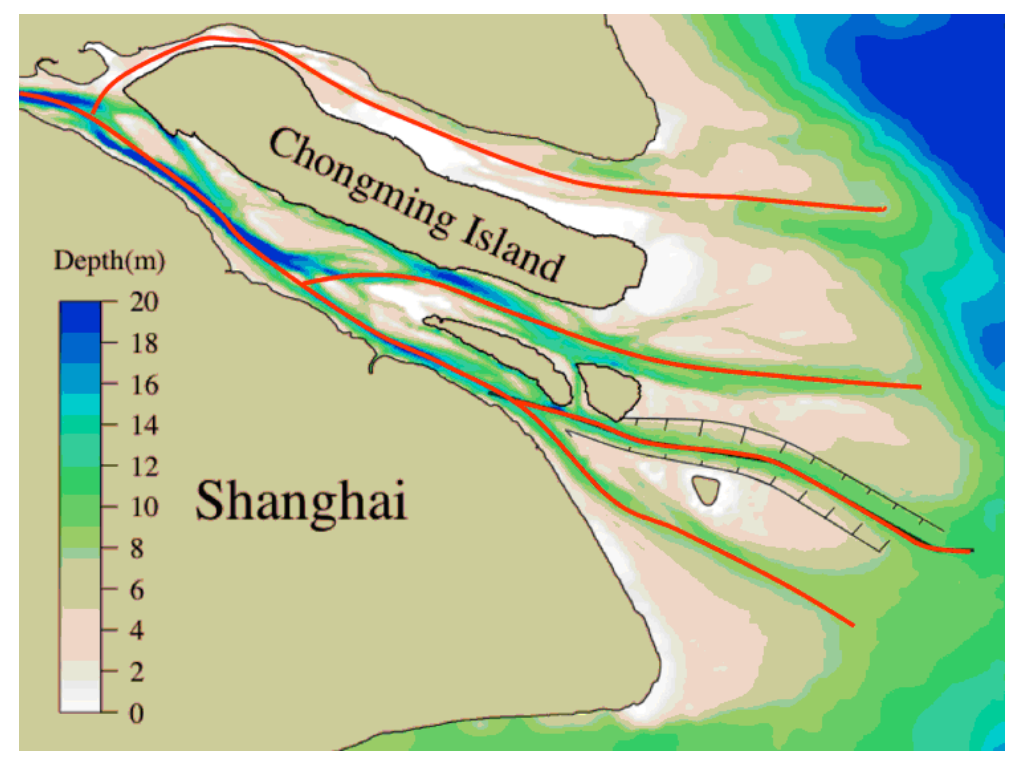

Figure 1. Yangtze Estuary with ordered branching channels.

The development of the system since the DNCP makes once more clear that it is important to improve our understanding of the behaviour of the bifurcating channel system in the estuary. For this purpose we present analysis on two aspects: (1) the equilibrium configuration of river delta distributary networks, and (2) influence of tidal flow on the morphological equilibrium of rivers. The analyses are mainly theoretical but supported by field data.

\section{RIVER DOMINATED DISTRIBUTARY DELTA NETWORKS}

\section{Regime theory and field observations}

The ordered bifurcating structure is a typical feature of the distributary channel networks in a non-tidal river delta, See Figure 2. For a single channel in such a system regime relations exist relating the dimensions of the channel to the discharge through the channel.

$$
W=a Q^{b} \quad D=c Q^{f}
$$

Each time a channel bifurcates the two downstream channels receives on average each the half of the discharge of the channel upstream. Therefore the dimensions of the channels of different order in the network follow the following relations:

$$
\frac{W_{n}}{W_{0}}=\frac{1}{2^{b n}} \text { and } \frac{D_{n}}{D_{0}}=\frac{1}{2^{f n}}
$$

Herein $a, b, c$ and $f$ are constant coefficients and further $W=$ width of a channel, $D=$ depth of a channel, $Q=$ discharge through a channel, $n=$ order of bifurcation (see Figure 2). Mikhalov (1970): $b=0.5$ and $f=0.33$, or

$$
\frac{W_{n+1}}{W_{n}} \approx 0.71 \& \frac{D_{n+1}}{D_{n}} \approx 0.80
$$

Andren (1994) reports $b=0.39 \& \mathrm{f}=0.383$, or

$$
\frac{W_{n+1}}{W_{n}} \approx 0.76 \& \frac{D_{n+1}}{D_{n}} \approx 0.77
$$


Edmonds and Slingerland (2007) report an analysis of data observed from 11 deltas in the world. The data include network structures, lengths and widths of the bifurcating channels. For three of the deltas bathymetric data are also collected so that information about channel depths can be obtained. The data analysis show that channel width, channel depth and channel length show well-defined decrease with bifurcation order. The data concerning the channel width and channel depths agree well with the relation derived from the regime relations of Andren (1994). Also the channel length shows a well-defined decrease with bifurcation order, but this cannot be explained by any known relations. Edmonds and Slingerland (2007) found that the aspect ratio of the channels is more or less constant for all the channels in the different bifurcation orders, or in other words the $b$ and $f$ have the same value. They further reports that for the pairs of arms up to $5^{\text {th }}$ order, the widths are not equal but occur most frequently in the ratio 1.7:1 (see Figure 3). It is interesting to note another feature of the networks which has not been mentioned by the authors: The larger channel usually bifurcates once more after a bifurcation than the smaller one.

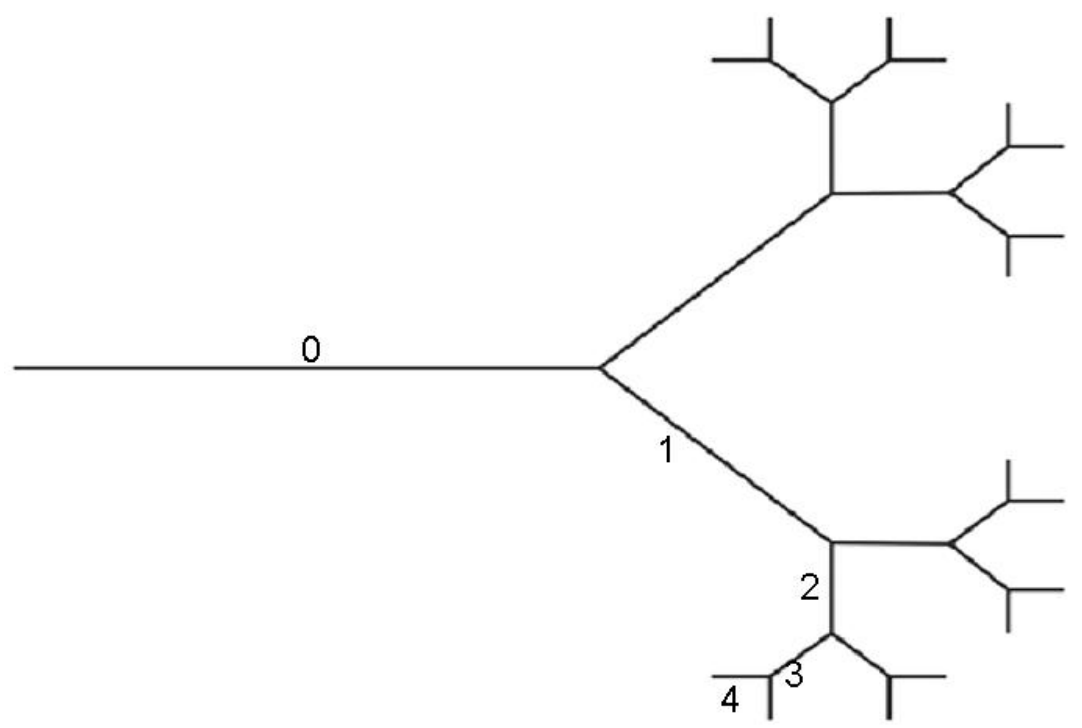

Figure 2. Schematic sketch of the bifurcating channel network in a river delta.

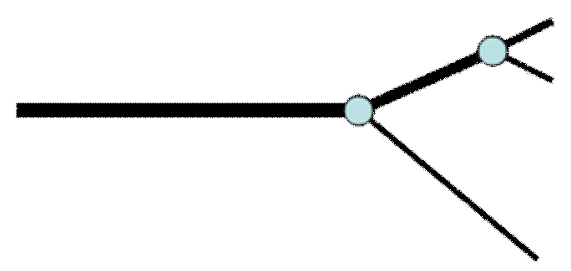

Figure 3. Schematic sketch of the asymmetric bifurcations.

\section{Theoretical analysis}

For a single bifurcation as sketched in Figure 4 the following equations are available for determining the equilibrium configurations according to the process-based 1D network morphodynamic model (Wang et al, 1995):

Mass-balance for water:

$$
Q_{1}+Q_{2}=Q_{0}
$$

Water motion described by Chezy formula: 


$$
Q_{j}=W_{j} C_{j} D_{j}^{\frac{3}{2}} i_{j}^{\frac{1}{2}} \quad j=1,2
$$

Geometric relation:

$$
i_{1} L_{1}=i_{2} L_{2}
$$

Mass-balance for sediment:

$$
S_{1}+S_{2}=S_{0}
$$

Sediment transport described by a power-law:

$$
S_{j}=W_{j} m\left(\frac{Q_{j}}{W_{j} D_{j}}\right)^{N} \quad j=1,2
$$

Nodal point relation for sediment distribution:

$$
\frac{S_{1}}{S_{2}}=\left(\frac{Q_{1}}{Q_{2}}\right)^{k}\left(\frac{W_{1}}{W_{2}}\right)^{1-k}
$$

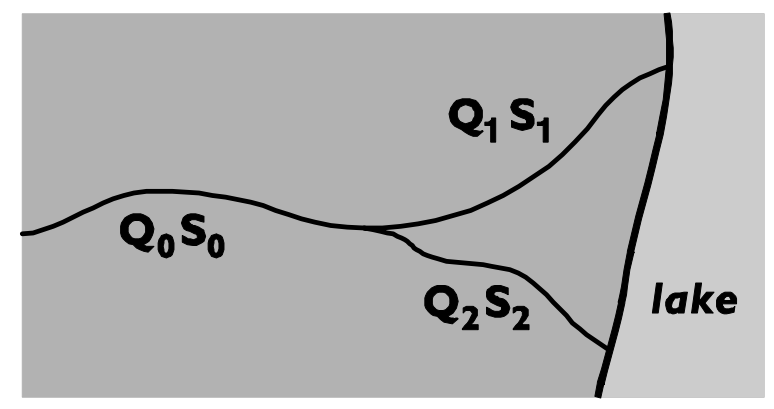

Figure 4. Schematic sketch of the asymmetric bifurcations.

Wang et al (1995) assumed that the geometric configuration on the horizontal plane is known and fixed. This means that the lengths and the widths of the channels are known. Then these eight equations are exactly sufficient for determining the eight unknown variables $Q_{j}, S_{j}, D_{j}$, and $i_{j}, j=1,2$. It is noted that the subscript ${ }_{1}$ and ${ }_{2}$ are in this section used for indicating the two branches downstream of the bifurcation, not for the order of bifurcations as in the previous section.

The assumption no more hold for the cases considered here. If we no more assume that the widths of the channels are given, two extra unknown variables are introduced. If we no more assume that the lengths of the channels are known, Eq.(3) can then no more be applied, i.e. another freedom is introduced. The consequences of these extra freedoms for determining the equilibrium configuration are considered here in two steps.

First consider case that the widths of the channels are unknown instead of known, but keep the assumption that the lengths are known. Two extra equations are then needed to complete the system of equations. In this case it is then logical to use the observation by Edmonds and Slingerland (2007) that the aspect ratio of the channels seems to be constant for all channels in the networks:

$$
\frac{W_{j}}{D_{j}}=\alpha
$$

Herein $\mathrm{a}$ is the known aspect ratio. Consider first a symmetric bifurcation, i.e. the two downstream branches are of the same length, 


$$
\begin{aligned}
& Q_{1}=Q_{2}=\frac{Q_{0}}{2} \\
& S_{1}=S_{2}=\frac{S_{0}}{2}
\end{aligned}
$$

The solution becomes:

$$
\begin{aligned}
& \frac{D_{1}}{D_{0}}=\frac{D_{2}}{D_{0}}=\left(\frac{1}{2}\right)^{\frac{N-1}{2 N-1}} \\
& \frac{i_{1}}{i_{0}}=\frac{i_{2}}{i_{0}}=\left(\frac{1}{2}\right)^{\frac{3-N}{2 N-1}}
\end{aligned}
$$

This also gives an explanation why the depths (therefore also the widths) of the channels decreases with the order of bifurcations. The relations of Andren (1994) agrees approximately with Eq.(13) for N=3. According to Eq.(13) the slopes of the channels will be the same for all orders of bifurcation if $\mathrm{N}=3$, and they increase with the order of bifurcations for $\mathrm{N}>3$.

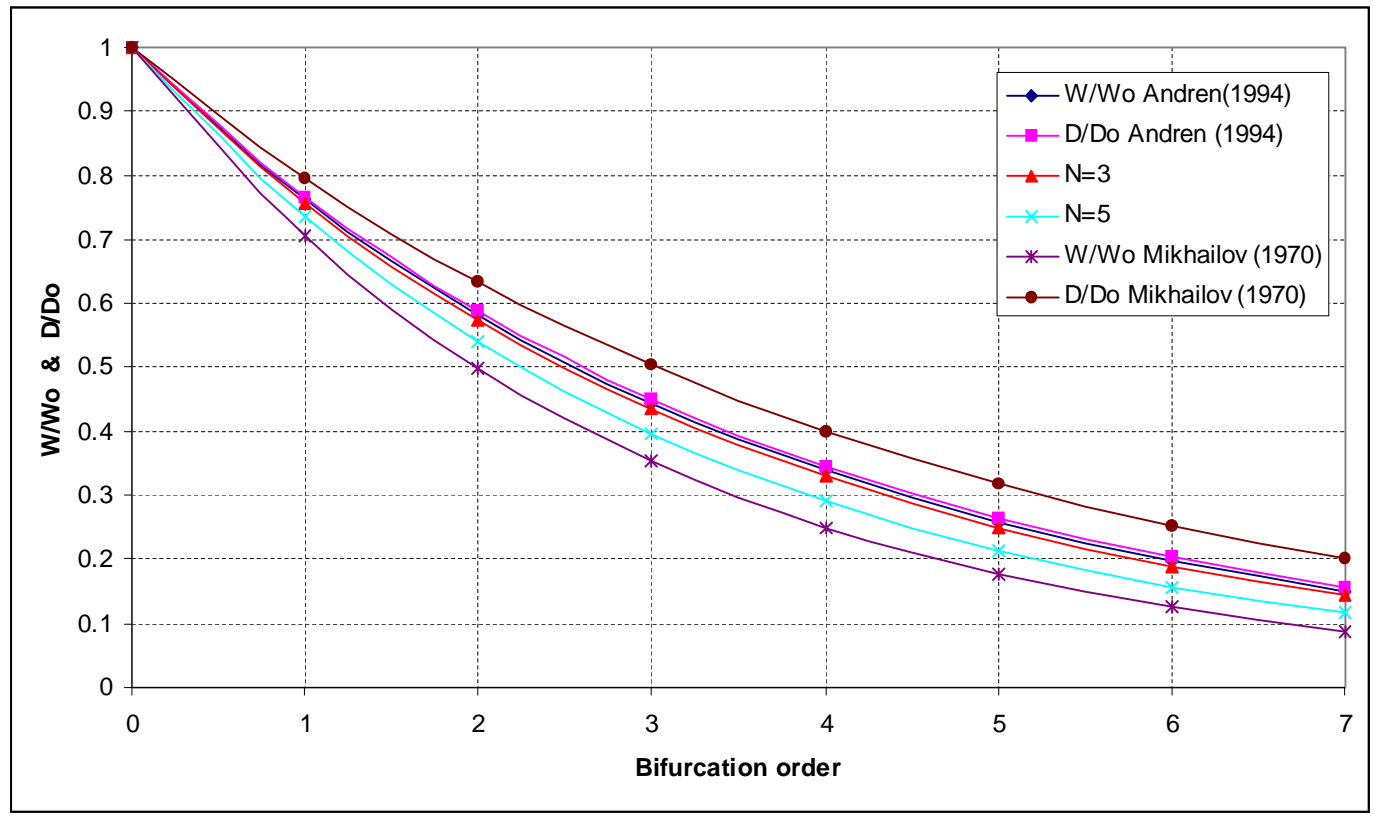

Figure 5. Analytical solution for networks with symmetric bifurcations.

In Figure 5 the analytical solutions for two different values of $\mathrm{N}$ are compared with the regimerelations. Note that the only model parameter having influence on the analytical solution is $\mathrm{N}$, the power in the sediment transport formula. Although the sediment transport rate is very sensitive to the power $\mathrm{N}$ the morphological equilibrium is much less sensitive to this parameter. The analytical solution is thus robust and explains the observed behaviour of the networks well.

Now also introduce the other freedom, the lengths of the channels are no more fixed. This has the consequence that another equation is required to replace Eq.(7). Here use is made of the observed constant width ratio to complete the system of equations.

$$
\frac{W_{1}}{W_{2}}=\beta
$$

Because of the constant aspect ratio for all channels we also have 


$$
\frac{D_{1}}{D_{2}}=\beta
$$

The solution becomes then:

$$
\begin{aligned}
& \frac{D_{1}}{D_{0}}=\beta\left(\frac{1+\beta^{\frac{K N+N-K}{N-K}}}{\left(1+\beta^{\frac{2 N-k}{N-k}}\right)^{N}}\right)^{\frac{1}{2 N-1}} \\
& \frac{D_{2}}{D_{0}}=\left(\frac{1+\beta^{\frac{K N+N-K}{N-K}}}{\left(1+\beta^{\frac{2 N-k}{N-k}}\right)^{\frac{1}{2 N-1}}}\right)^{\left.\frac{i^{N}}{\left(1+\beta^{\frac{2 N-k}{N-k}}\right.}\right)^{\frac{N+2}{2 N-1}}} \\
& \frac{i_{1}}{i_{0}}=\beta^{\frac{3 k-N}{N-k}} \frac{\left(1+\beta^{\frac{K N+N-K}{N-K}}\right.}{\left(1+\beta^{\frac{5}{2 N-k}}\right.} \\
& \frac{i_{2}}{i_{0}}=\frac{\left(1+\beta^{\frac{K N+N-K}{N-K}}\right)^{\frac{N+2}{2 N-1}}}{\left(1+\frac{5}{2 N-1}\right.}
\end{aligned}
$$

The analytical solution for the morphological equilibrium depends now in addition on the power $\mathrm{N}$ in the sediment transport formula also on the power $\mathrm{k}$ in the nodal point relation. It can again be shown that the solution for the width and depth is not very sensitive to $\mathrm{N}$. The sensitivity of the solution to $\mathrm{k}$ is shown in Figure 6. It is concluded that also for the asymmetric case the analytical solution is robust. It is also observed that the ratio between the averaged value of the widths of the two downstream braches to the width of the upstream branch is about the same as according to the solution for the symmetric case and thus agrees well with the observations.

In Figure 6 the solutions for the bed slope are shown as well. For the symmetric case, the bed slope of the downstream branches is larger than that of the upstream branch. This applies also for the larger downstream branch in the asymmetric case. The slope of the smaller downstream branch is larger than that of the larger branch for $\mathrm{k}$ smaller than the critical value for stability of the bifurcation, and for $\mathrm{k}$ larger than the critical value the slope of the smaller branch is smaller than that of the larger branch. In other words, for stable bifurcations the larger branch has larger bed slope. 

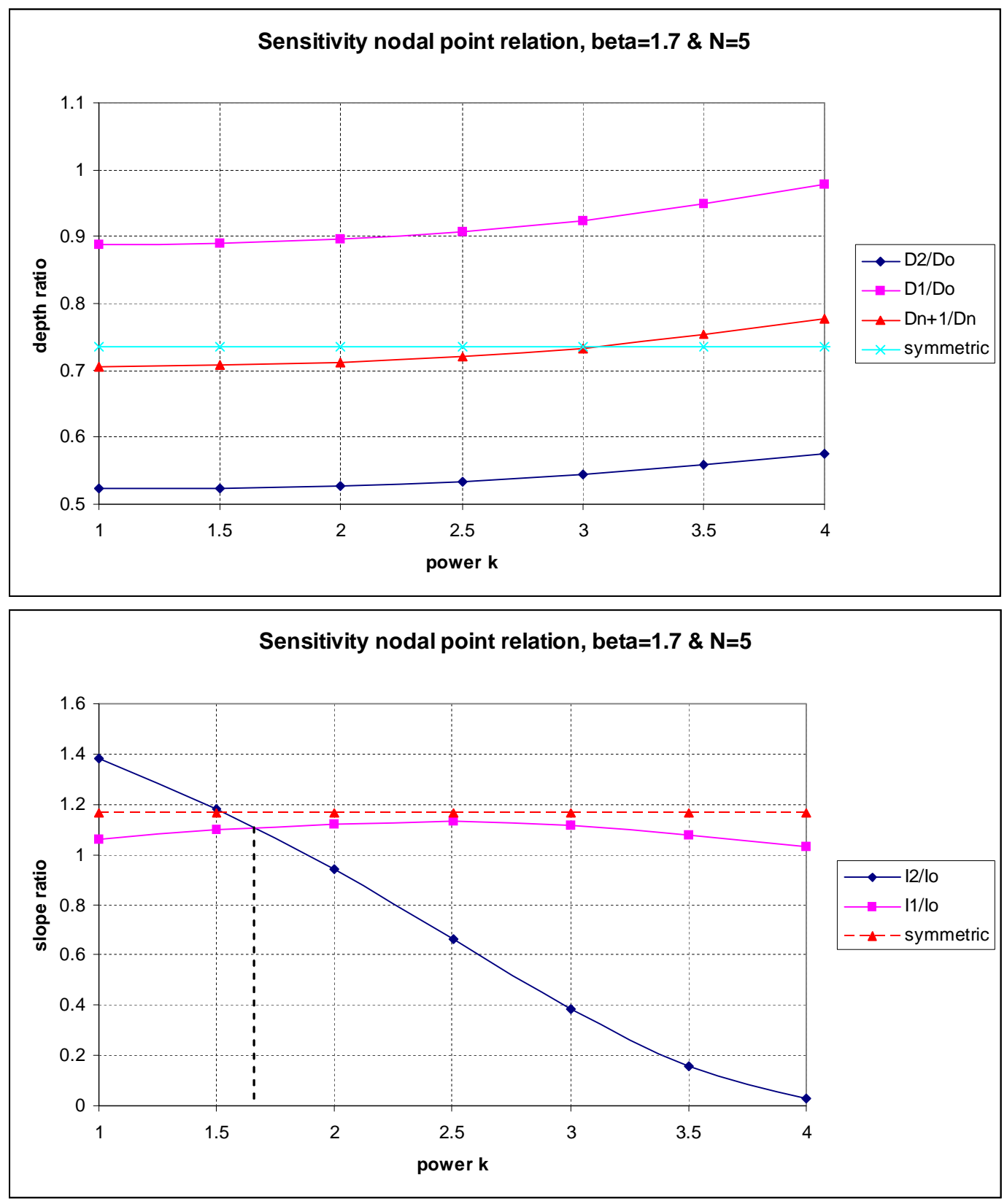

Figure 6. Sensitivity of the analytical solution to the power $\mathbf{k}$.

\section{INFLUENCE OF TIDE}

The channel network structure in the Yangtze Estuary (Figure 1) has the same basic structure as the distributary channel networks in a non-tidal river delta. The ordered bifurcations show asymmetric characteristics, the same as a non-tidal river delta as described by Edmonds and Slingerland (2007). This reveals the relative importance of the fluvial influences to this estuary. However, there are also some differences between the Yangtze Estuary and a non-tidal river delta concerning the channel network structure. The Yangtze Estuary has only three levels of bifurcations whereas Edmonds and Slingerland (2007) report up to 7 levels of bifurcations in non-tidal river deltas. Another difference is that each channel in the Yangtze Estuary shows a downstream widening which is not the case in a non-tidal river delta. According to the analysis in the previous chapter, the bed slope of the channels in the non-tidal river delta show an increasing trend in the downstream direction. This is clearly opposite to the situation in the Yangtze Estuary. In fact, increasing width and decreasing bed slope 
are common features of most estuaries. In the analysis below we show that this is due to the influences of the tide.

We start from the 1D model which is often used for rivers. The following equations are available:

$$
\begin{gathered}
W \frac{\partial \varsigma}{\partial t}+\frac{\partial Q}{\partial x}=0 \\
\frac{\partial Q}{\partial t}+\frac{\partial}{\partial x}\left(\gamma \frac{Q^{2}}{A}\right)+g A \frac{\partial \varsigma}{\partial x}+\frac{g Q|Q|}{C^{2} A D}=0 \\
S=W m\left(\frac{Q}{A}\right)^{N} \\
W \frac{\partial z_{b}}{\partial t}+\frac{\partial S}{\partial x}=0
\end{gathered}
$$

In these equations $\mathrm{Z}=$ water level, $t=$ time, $x=$ horizontal coordinate along the river, $\mathrm{g}=$ coefficient, $A=$ cross-sectional area for stream, $g=$ gravitational acceleration.

For non-tidal rivers the morphological equilibrium can be derived by requiring that all time derivatives are zero. For the simplest case, steady-uniform flow, the equations reduce to those for a single branch as presented in the previous chapter (Eqs. 6 and 9). These two equations determine the two parameters describing the morphology, depth $D$ and slope $i$ for given width $W$. Physically it means that the river adjust its depth and slope such that the water and sediment from the river catchment can exactly be discharged and transported to downstream.

For the tidal river case the requirement for morphological equilibrium should be changed from 'all variables are constant, or their time-derivatives are zero' to 'all variables are periodic'. Derivation of the morphological equilibrium is no more straightforward if possible. Earlier works even question the existence of an equilibrium. Here we only show the effects of the fluctuation of the flow on the two basic functions of a river, i.e. discharging water and transporting sediment into the sea. The basic thought is thus the same as in the case of non-tidal rivers: the morphology should adjust such that the residual discharge and the residual sediment transport through each cross-section are equal to those supplied from the upstream river. Formal integrations of the equations (17) to (20) yields:

$$
\begin{gathered}
\bar{Q}=\text { constant }=Q_{0} \\
I=\frac{\partial \bar{\zeta}}{\partial t}=\frac{1}{T} \int_{0}^{T} \frac{Q|Q|}{C^{2} A^{2} D} d t-\frac{1}{T} \int_{0}^{T} \frac{1}{g A} \frac{\partial}{\partial x}\left(\alpha \frac{Q^{2}}{A}\right) d t \\
\bar{S}=\frac{1}{T} \int_{0}^{T} W m\left(\frac{Q}{A}\right)^{N} d t \\
\bar{S}=\text { constant }=S_{0}
\end{gathered}
$$

In these equations the over bar denotes tidal averaging, $T$ is tidal period and the subscript ${ }_{0}$ represents the upstream (non-) tidal river value. Now we make the following simplifying assumptions:

- Amplitude of the water level fluctuation is much smaller than the water depth.

- The advection term, i.e. the last term in equation (22), can be neglected.

Under these assumptions the equations (22) and (23) become similar as in the case of non-tidal rivers. 


$$
\begin{gathered}
C^{2} A^{2} D I=\frac{1}{T} \int_{0}^{T} Q|Q| d t \text { or } Q_{e f}=A C \sqrt{D I} \\
\bar{S}=\frac{W m}{A^{n}} \frac{1}{T} \int_{0}^{T} Q^{N} d t=W m\left(\frac{Q_{e s}}{A}\right)^{N}
\end{gathered}
$$

The difference is that the constant upstream river discharge can not be used but should be replaced by an effective discharge for respectively the resistance term and for the sediment transport.

$$
\begin{gathered}
Q_{e f}=\sqrt{\frac{1}{T} \int_{0}^{T} Q|Q| d t} \\
Q_{e s}=\sqrt[N]{\frac{1}{T} \int_{0}^{T} Q^{n} d t}
\end{gathered}
$$

Note that both effective discharges are larger than the upstream river discharge $Q_{0}$. Physically it means that the fluctuation of the flow increases the resistance for the river to discharge the water to the sea and that the fluctuation of the flow increases the sediment transport capacity of the river. These two effects have influence on the morphological equilibrium of the tidal section of a river. To illustrate this we consider the wide river case. The equations determining the morphological equilibrium become:

$$
\begin{gathered}
Q_{e f}=W C D^{\frac{3}{2}} I^{\frac{1}{2}} \\
m W^{N-1} D^{N}=\frac{Q_{e s}^{N}}{S_{0}}
\end{gathered}
$$

Combining with the equations for the upstream non-tidal part of the river yields:

$$
\begin{gathered}
\frac{W}{W_{0}}\left(\frac{D}{D_{0}}\right)^{\frac{3}{2}}\left(\frac{I}{i_{0}}\right)^{\frac{1}{2}}=\frac{Q_{e f}}{Q_{0}} \\
\left(\frac{W}{W_{0}}\right)^{N-1}\left(\frac{D}{D_{0}}\right)^{N}=\left(\frac{Q_{e s}}{Q_{0}}\right)^{N}
\end{gathered}
$$

These equations make it clear that the slope of the river becomes smaller and the cross-section becomes larger (wider and/or deeper) in the downstream direction due to increased tidal influence. Note that another difference with the non-tidal river is that the width $W$ surely also need to be considered as unknown. So there are two equations for three variables. For non-tidal we often assume that $W$ is given and $D$ and $i$ can be solved from the two equations. There is one exception for the case that $n=3$, then $I$ can be solved from (31) and (32):

$$
\frac{I}{i_{0}}=\left(\frac{Q_{e f}}{Q_{0}}\right)^{2}\left(\frac{Q_{e s}}{Q_{0}}\right)^{-N}
$$


We now make another simplifying assumption, just to show the effect of tidal fluctuation: The tidal fluctuation can be represented by a single harmonic function. In other words, only one tidal component is considered, or

$$
Q=Q_{0}+\hat{Q} \cos \left(\frac{2 \pi t}{T}-\varphi\right)
$$

Both the amplitude and the phase of the tidal variation part are function of $x$. Equation (27) gives for the river section upstream of the tidal flow limit $(Q>0)$ :

$$
Q_{e f}=\sqrt{Q_{0}^{2}+\frac{\hat{Q}^{2}}{2}}
$$

And Eq.(28) gives for $n=3$

$$
Q_{e s}=\sqrt[3]{Q_{0}^{3}+\frac{3}{2} Q_{0} \hat{Q}^{2}}
$$

For $n=5$

$$
Q_{e s}=\sqrt[3]{Q_{0}^{5}+5 Q_{0}^{3} \hat{Q}^{2}+\frac{15}{8} Q_{0} \hat{Q}^{4}}
$$

At the tidal flow limit we have per definition

$$
\hat{Q}=Q_{0}
$$

So for $n=3$

$$
\begin{gathered}
\frac{I}{i_{0}}=\frac{3}{5} \\
\left(\frac{A}{A_{0}}\right)^{2} \frac{h}{h_{0}}=\frac{5}{2}
\end{gathered}
$$

For $n=5$

$$
\begin{aligned}
& \left(\frac{A}{A_{0}}\right)^{2} \frac{h}{h_{0}} \frac{I}{i_{0}}=\frac{3}{2} \\
& \left(\frac{A}{A_{0}}\right)^{4} \frac{h}{h_{0}}=\frac{63}{8}
\end{aligned}
$$

With an extra assumption e.g. that $\mathrm{h}$ is constant we obtain for $\mathrm{n}=3$

$$
\begin{gathered}
\frac{I}{i_{0}}=\frac{3}{5} \\
\frac{A}{A_{0}}=\frac{B}{B_{0}}=\sqrt{\frac{5}{2}} \approx 1.58
\end{gathered}
$$

For $n=5$ 


$$
\begin{gathered}
\frac{I}{i_{0}}=\frac{3}{2} \sqrt{\frac{8}{63}} \approx 0.53 \\
\frac{A}{A_{0}}=\frac{B}{B_{0}}=\sqrt[4]{\frac{63}{8}} \approx \sim 1.68
\end{gathered}
$$
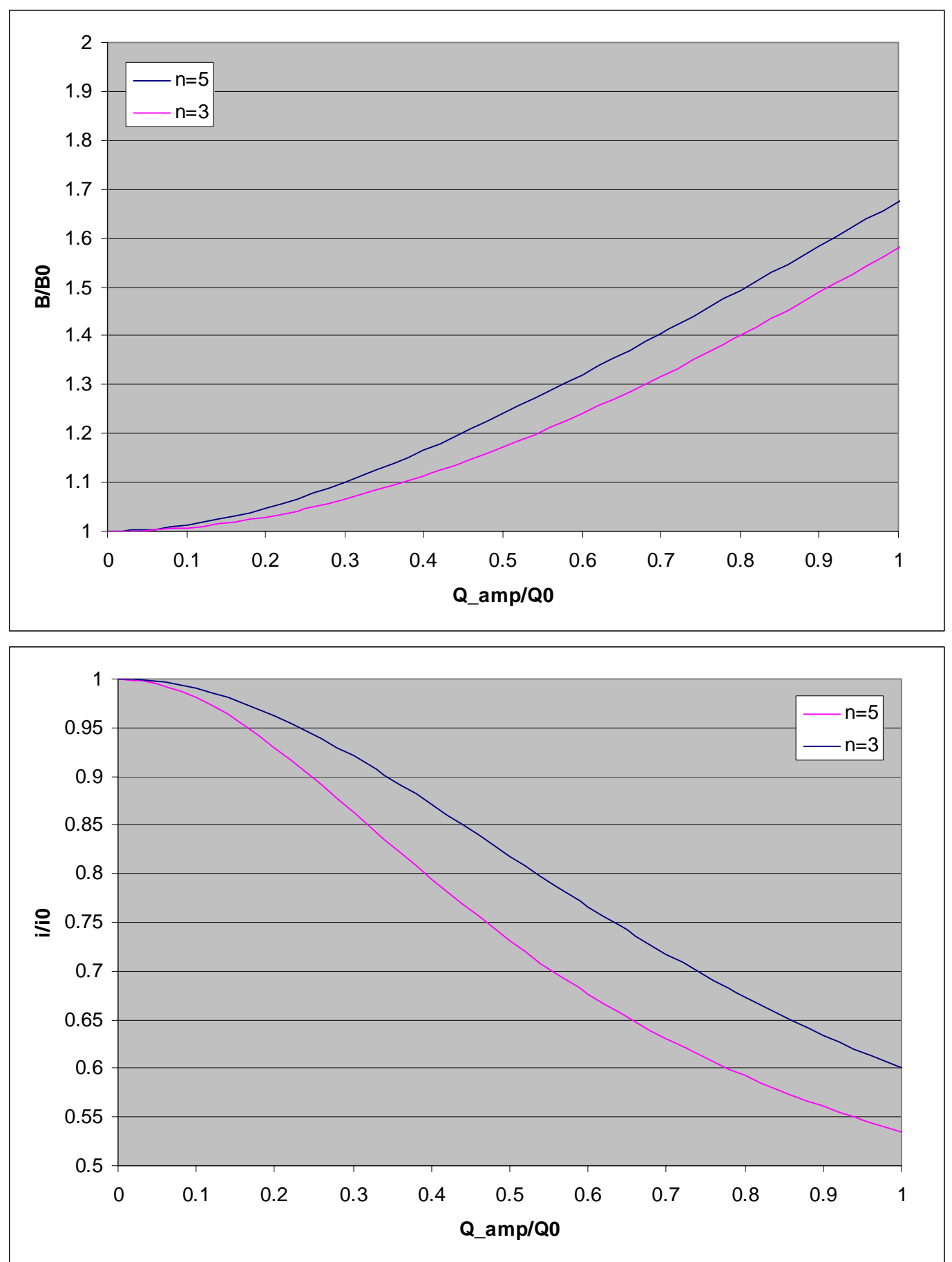

Figure 7. Equilibrium depth and equilibrium slope as function of the tidal amplitude in the region between tidal limit and tidal flow limit: 0 on the horizontal axis represents the non-tidal part of the river and 1 represents the tidal flow limit of an estuary. 
These results are shown in Figure 7 for the river section between the tidal limit and the tidal flow limit, and appear to be not very sensitive to the value of $N$. The results of the analysis show thus that the tidal influences explain why the channels in estuaries show increasing width and decreasing slope in the downstream direction.

It is noted that the region between the tidal limit and the tidal flow limit is often not a negligible section of the river. For the Yangtze this is from Datong to Jianyin, several hundreds km long. Measured on Google Earth, the width at the narrowest section near Datong is about $1250 \mathrm{~m}$, and near Jianyin about $2000 \mathrm{~m}$. The ratio between the widths of these two cross-sections is thus about 1.6, which agrees well with the results shown in Figure 7. Downstream of the tidal limit the analysis can also be applied, but then there are many complications: salt intrusion, bifurcation of the river / estuary, etc.

\section{CONCLUSIONS}

The following conclusions are drawn from the analyses in the previous two chapters:

- The branching channel structure of the Yangtze Estuary can be classified as tide-influenced river delta distributary networks. Its basic structure is the same as in case of river dominated delta (see e.g. Edmonds and Slingerland, 2007), but there are also differences between the Yangtze Estuary case and the non-tidal river delta.

- The empirical relations, describing the basic features of the river-dominated distributary delta networks, can be explained by theoretical analysis.

- Not all the rules governing the features of the river-dominated delta networks are satisfied by the Yangtze Estuary. The most important deviations can mainly be explained by the influences of the tide.

- The tidal influence can be analysed theoretically. Two major influences of the tide are identified, viz. increasing the resistance to the river flow into the sea and increasing the sediment transport capacity. As consequence of these two influences the cross-sectional area of the river/estuary increases in the seawards direction and the bed slope decreases.

\section{ACKNOWLEDGMENTS}

This paper is a product of the project "Effect of human activities on eco-morphological evolution of rivers and estuaries" within the Programme of Strategic Scientific Alliance between China and The Netherlands (PSA), financed by Royal Dutch Academy of Sciences and Arts (KNAW), project nr. 08PSA-E-001, and the Chinese Ministry of Science and Technology (MOST), project nr. 2008DFB90240. The work is further supported by various projects at Deltares: The coastal research project finance by Rijkswaterstaat, The various Building with Nature projects financed by Ecoshape, and the Long-Term Vision Scheldt Estuary project.

\section{REFERENCES}

Andrén, H. (1994), Development of the Laitaure delta, Swedish lappland: A study of growth, distributary forms, and processes, Ph.D. thesis, 188 pp., Institute of Earth Sciences: Physical Geography, Uppsala University, Uppsala, Sweden.

Chen, J.Y., Yu, C.X. and Hu H.C., 1982, The model of development of the Changiiang estuary during the last 2000 years, In Kennedy, J.F. (editor), Estuarine comparison, Academic press, 1982, pp. 655-666.

Edmonds, D. A. \& Slingerland, R. L. Mechanics of river mouth bar formation: Implications for the morphodynamics of delta distributary networks. J. Geophys. Res. 112, F02034 (2007).

Mikhailov, V. N. (1970), Hydrologic-morphometric characteristics of delta branches, Studies and Reports in Hydrology 9, IASH/UNESCO, 146-158.

Wang, Z. B., M. De Vries, R. J. Fokkink, and A. Langerak (1995), Stability of river bifurcations in 1d morphodynamic models, J. Hydraul. Res. 33, 739-750. 